\title{
ROE y estructura financiera de las empresas mineras periodo 2004-2013 ${ }^{1}$
}

\section{ROE and financial structure of mining companies period 2004-2013}

\section{RESUMEN}

Raimundo Renaun Pacheco Mexzon ${ }^{2}$

El trabajo de investigación llamado "ROE y ESTRUCTURA FINANCIERA DE LAS EMPRESAS MINERAS PERIODO 2004-2013” se preparó tomando en cuenta el comportamiento y la importancia del sector minero dentro de la formación del producto bruto interno (PBI) del país. Hicimos las siguientes preguntas:

1. ¿Qué comportamiento tubo el rendimiento del patrimonio (ROE: Return on Equity) de las empresas peruanas del Sector Minero que cotizan en la Bolsa de Valores de Lima durante el periodo 2004 - 2013?

2. ¿Qué empresas optimizaron su estructura financiera?

Para responderlas se desarrollaron cinco capítulos. Se concluyó que las empresas presentan Estructura Financiera y ROE diferentes.

Palabras clave: Rentabilidad del patrimonio (ROE); estructura financiera.

JEL: G11, G32

1 La inquietud de plantear el artículo es divulgar los resultados obtenidos por el grupo de investigación al desarrollar el proyecto de investigación ROE y Estructura financiera de las Empresas Mineras Periodo 2004-2013. Al conocer el comportamiento del ROE de la empresa y su estructura financiera, podemos clasificar el artículo como de reflexión y debate.

2 Doctor en Economía por la Universidad Nacional Mayor de San Marcos. Ex Gerente Central de Finanzas y Administración de CENTROMIN PERÚ S.A. Ex Vicepresidente de MINPECO PERÚ S.A., MINPECO USA Y MINPECO UK. E-mail: rpachecom@unmsm.edu.pe.

(C) Los autores. Este artículo es publicado por Pensamiento Crítico de la Facultad de Ciencias Económicas, Universidad Nacional Mayor de San Marcos. Este es un artículo de acceso abierto, distribuido bajo los términos de la licencia Creative Commons Atribucion - No Comercia_Compartir Igual 4.0 Internacional. (http://creativecommons.org/licenses/by-nc-sa/4.0/) que permite el uso no comercial, distribución y reproducción en cualquier medio, siempre que la obra original sea debidamente citada. 


\section{ABSTRACT}

The research work called "ROE and FINANCIAL STRUCTURE OF MINING COMPANIES PERIOD 2004-2013" was developed after considering the following questions:

1. What has been the return on equity performance (ROE: Return on Equity) of the Peruvian mining sector companies that are listed on the Lima Stock Exchange during the 2004-2013 period?

2. Which companies in this sector of the Peruvian economy that are listed on the Lima Stock Exchange have optimized their financial structure?

To answer them, five chapters were developed and it was concluded that not all companies present the same profitability opportunities (ROE) and this differs according to the size of the company and the behavior of its financial structure.

Keywords: Return On Equity (ROE); financial structure.

JEL: G11, G32

\section{Contenido del informe en extenso de la investigación}

En el CAPÍTULO I, “Aspectos Metodológicos”, se desarrolló el marco teórico, el planteamiento del modelo que permite calcular la estructura optima de capital de una empresa, objetivos, hipótesis y la justificación de la investigación.

En el CAPÍTULO II, se determinó la muestra y se tomaron 20 empresas que pertenecen al sector minero. Posteriormente se ordenó a las empresas según su patrimonio para finalmente seleccionar a empresas grandes, medianas y pequeñas representativas que fueron objeto de estudio.

En el CAPÍTULO III, se realizó una descripción de los datos más relevantes de cada empresa seleccionada. Este capítulo permitirá al lector conocer el giro de negocio de la empresa, sus accionistas, el ingreso por ventas en los últimos años, entre otros datos que ayudará a entender mejor el desempeño de la empresa.

En el CAPÍTULO IV, aplicamos el modelo desarrollado en el marco teórico, calculamos el ROE y graficamos su evolución.

En el CAPÍTULO V, se realizó un breve análisis y se determinó que empresas tuvieron los mejores resultados durante el periodo de análisis. 


\section{Formulación del Problema}

Las empresas para evaluar inversiones que realizaran para crecer, utilizan como tasa de descuento el Costo del Capital Promedio Ponderado (Weighted Average Cost of Capital).

Una parte importante de este costo lo constituye el capital propio que está representado por el Patrimonio de la empresa el cual permite calcular la rentabilidad del patrimonio conocido como $\mathrm{ROE}^{3}$. Y la otra parte está formada por las deudas o pasivos. La sumatoria del patrimonio (P) y las deudas o pasivos (D) permiten calcular la estructura financiera de la empresa.

Tomando en cuenta lo planteado en el párrafo anterior, el trabajo de investigación se desarrolló luego de plantearnos las siguientes preguntas:

1. ¿Qué comportamiento tubo el rendimiento del patrimonio (ROE: Return on Equity) de las empresas peruanas del Sector Minero que cotizan en la Bolsa de Valores de Lima durante el periodo $2004-2013 ?$

2. ¿Qué empresas optimizaron su estructura financiera.

Para responderlas se desarrollaron cinco capítulos. Se concluyó que las empresas presentan Estructura Financiera y ROE diferentes.

\section{Marco Teórico}

\section{Rentabilidad del patrimonio (ROE)}

ROE (Return on Equity) es un índice de rentabilidad que relaciona la utilidad neta entre el patrimonio. Por ejemplo si en un año la empresa presenta en su estado financiero de Ganancias y Pérdidas una utilidad neta después de impuestos de US\$100 y en el Balance General un patrimonio de US\$1,000 se tiene un ROE de $10 \%$ (100 entre 1,000 = 0.10 ó 10\%). Este resultado nos indica que el patrimonio de la empresa genera un rendimiento del $10 \%$.

3 Rentabilidad del patrimonio: ROE (Return On Equity) 
Si el patrimonio hubiera sido mayor por ejemplo US\$1,500 el ROE disminuye llegando a $6.66 \%$ (100 entre 1,500 = 0.0666 ó $6.66 \%$ ). Si el patrimonio hubiera sido menor por ejemplo US\$ 500 el ROE aumenta llegando a $20 \%$ (100 entre $500=0.20$ ó $20 \%$ ).

Se observa que al mantener la utilidad neta en US\$100 se puede hacer crecer o disminuir la rentabilidad del patrimonio (ROE) dependiendo del patrimonio que tenga una empresa.

En consecuencia se puede maximizar el ROE si se tiene un nivel adecuado de patrimonio. Esto nos indica que el Gerente Financiero de una empresa debe preocuparse de mantener un nivel adecuado de patrimonio.

La fórmula para calcular el ROE es ${ }^{4}$ :

$$
R O E=\frac{U t . N e t}{P}
$$

Donde:

ROE $=$ Rentabilidad del patrimonio

Ut.Net $=$ Utilidad neta

$\mathrm{P}=$ Patrimonio

\section{Estructura financiera o de capital}

Hablar de la estructura financiera o de capital de una empresa es referirse a como está financiado el total de activos (propiedades y derechos adquiridos de la empresa). Por lo general el total de activos están financiados con deudas y patrimonio.

Para determinar la estructura financiera o de capital de la empresa se parte de la siguiente fórmula ${ }^{5}$ :

Donde:

$$
\text { Act.To. }=D+P
$$

Act. To. $=$ Activo Total

$\mathrm{D}=$ Deudas o pasivos

$\mathrm{P}=$ Patrimonio o recursos propios

\footnotetext{
4 Forsyth Alarco Juan Alberto. Finanzas empresariales: Rentabilidad y Valor. 2004. Pag59.

5 Bolten Steven E. Manual de Administración Financiera Volumen 1. 1987 pag 119
} 
Si dividimos la igualdad (2) Act.To $=\mathbf{D}+\mathbf{P}$ entre Act. To, tenemos lo siguiente:

$$
\frac{\text { Act. To }}{\text { Act. To }}=\frac{D+P}{\text { Act. To }}
$$

Desagregando la igualdad (3) tenemos:

$$
\frac{\text { Act. To }}{\text { Act. To }}=\frac{D}{\text { Act. To }}+\frac{P}{\text { Act. To }}
$$

Como Act. To es igual a $\mathbf{D + P}$ la ecuación anterior queda de la siguiente manera:

$$
\frac{(D+P)}{(D+P)}=\frac{D}{(D+P)}+\frac{P}{(D+P)}
$$

Desarrollando tenemos:

$$
I=\frac{D}{(D+P)}+\frac{P}{(D+P)}
$$

Pero como 1 es igual al $100 \%$ la igualdad anterior queda de la siguiente manera:

$$
100 \%=\frac{D}{(D+P)}+\frac{P}{(D+P)}
$$

Esta última ecuación nos indica que la deuda y el patrimonio son un porcentaje del activo total.

Por ejemplo si en una empresa el total de activos llega a US\$ 3.000, los pasivos o deudas llegan a US\$ 900 y el patrimonio a US\$ 2.100; la estructura financiera o de capital de esta empresa será:

$$
\begin{aligned}
& 100 \%=\frac{900}{3.000}+\frac{2,100}{3.000} \\
& 100 \%=0,30+0,70 \\
& 100 \%=30 \%+70 \%
\end{aligned}
$$


Esto nos indica que la estructura financiera de la empresa es $30 \%$ de deuda y $70 \%$ de patrimonio.

Dicho de otra manera, el total de activos de la empresa que es el 100 $\%$ está financiado $30 \%$ con pasivos o deudas a terceros y $70 \%$ con recursos propios o patrimonio de la empresa.

\section{Objetivos de la Investigación}

\section{Objetivo General}

Determinar si las empresas del Sector Minero que cotizan en la Bolsa de Valores de Lima tuvieron buenos resultados económicos en función a una estructura optima de capital en el periodo $2004-2013$.

\section{Objetivos Específicos}

1. Determinar si las empresas minero metalúrgicas que cotizan en la Bolsa de Valores de Lima tuvieron ROE constante o fluctuante.

2. Determinar qué empresas del sector minero tuvieron los mejores resultado y cuál fue su estructura de capital que maximiza el ROE.

\section{Justificación de la Investigación}

- Nuestra investigación tiene una justificación práctica ya que servirá como fuente de consulta académica para quienes busquen evaluar las futuras inversiones en el sector minero de nuestro país, y también para futuras extensiones en el tema que utilicen la rentabilidad del patrimonio y la estructura óptima de capital como insumo para nuevas investigaciones.

- Otro motivo que nos impulsa a realizar el presente trabajo es el descubrir que empresas mineras metalúrgicas tuvieron estructura financiera adecuada durante los años 2004 - 2013.

- El maximizar el ROE con una estructura óptima de capital es de gran importancia en la evaluación de proyectos de inversión y teniendo en cuenta que en el país las empresas constantemente to- 
man decisiones de inversión, nace la inquietud de analizarlas en el periodo 2004-2013 cuando se obtiene la mayor rentabilidad.

\section{De la Investigación}

- Tipo de Estudio

En la etapa inicial el estudio parte como exploratorio, debido a la necesidad de indagar en el campo de las finanzas y buscar antecedentes en trabajos anteriores sobre la rentabilidad del patrimonio y la estructura óptima de capital. Posteriormente el estudio se torna descriptivo durante la medición del cálculo del ROE y finalmente correlacional al mostrar la relación entre el ROE y la estructura de capital de la empresa.

\section{- Tipo de la Investigación}

El tipo de investigación es no experimental pues no se alterarán las variables independientes y porque es una investigación expost. Asimismo el diseño a usar es el longitudinal de panel, ya que analizaremos cambios a través del tiempo (2004-2013) sin manipular los datos que presentan las empresas.

Hay que tener en cuenta que nuestro estudio será inductivo ya que partiremos del análisis de un estudio particular (empresas seleccionadas del Sector Minero que cotizan en la Bolsa de Valores de Lima) a un análisis general para el sector.

\section{Formulación de Hipótesis}

- Hipótesis de Encuadramiento

El máximo ROE con una adecuada estructura de capital es una buena herramienta para evaluar la rentabilidad de la empresa porque es un importante indicador de la rentabilidad de los accionistas.

- Hipótesis Específicas

1. Las empresas analizadas han presentado una rentabilidad del patrimonio fluctuante. 
2. Las grandes empresas mineras tuvieron los mejores rendimientos de su patrimonio y mejor estructura de capital.

\section{Cálculo de la rentabilidad del patrimonio y la estructura de capital de las empresas seleccionadas}

Para cada empresa se hizo el análisis que se presenta a continuación. Se toma como ejemplo lo que se presenta en el informe en extenso para la Sociedad Minera Cerro Verde como gran empresa, Shougang Hierro Perú como mediana empresa y Compañía Minera Raura como pequeña empresa:

\section{Sociedad Minera Cerro Verde S.A.A}

Con estos datos históricos del activo, pasivo y patrimonio se calculó el ROE y la estructura financiera. Los resultados se muestran a continuación en el Cuadro $n^{\circ} 1$ y Gráfico $n^{\circ} 1$ :

Cuadro n ${ }^{\circ}$. ROE y Estructura de Capital de la SOCIEDAD MINERA CERRO VERDE S.A.A.

\begin{tabular}{|c|c|c|c|c|c|c|c|c|c|c|}
\hline COEFICIENTE & $\mathbf{2 0 0 4}$ & $\mathbf{2 0 0 5}$ & $\mathbf{2 0 0 6}$ & $\mathbf{2 0 0 7}$ & $\mathbf{2 0 0 8}$ & $\mathbf{2 0 0 9}$ & $\mathbf{2 0 1 0}$ & $\mathbf{2 0 1 1}$ & $\mathbf{2 0 1 2}$ & $\mathbf{2 0 1 3}$ \\
\hline ACTIV0 & $1,015,680$ & $2,410,819$ & $4,607,004$ & $5,540,410$ & $5,518,344$ & $5,337,728$ & $6,400,478$ & $8,918,506$ & $11,379,163$ & $13,470,681$ \\
\hline PASIV0 & 274,625 & 196,628 & $1,088,655$ & $1,506,790$ & $1,823,896$ & $1,303,128$ & $1,938,601$ & $1,447,896$ & $1,686,083$ & $2,066,600$ \\
\hline PATRIMONI0 & 741,055 & $2,214,191$ & $3,518,349$ & $4,033,620$ & $3,694,448$ & $4,034,599$ & $4,461,877$ & $7,470,610$ & $9,693,079$ & $11,404,080$ \\
\hline UTILIDAD NETA & 244,580 & 651,270 & $1,240,493$ & $2,245,071$ & $2,004,428$ & $1,976,793$ & $2,997,556$ & $3,008,733$ & $2,222,469$ & $1,711,001$ \\
\hline ROE & $33.00 \%$ & $29.41 \%$ & $35.26 \%$ & $55.66 \%$ & $54.26 \%$ & $49.00 \%$ & $\mathbf{6 7 . 1 8} \%$ & $40.27 \%$ & $22.93 \%$ & $15.00 \%$ \\
\hline K & $72.96 \%$ & $91.84 \%$ & $76.37 \%$ & $72.80 \%$ & $66.95 \%$ & $75.59 \%$ & $\mathbf{6 9 . 7 1 \%}$ & $83.77 \%$ & $85.18 \%$ & $84.66 \%$ \\
\hline W & $27.04 \%$ & $8.16 \%$ & $23.63 \%$ & $27.20 \%$ & $33.05 \%$ & $24.41 \%$ & $\mathbf{3 0 . 2 9} \%$ & $16.23 \%$ & $14.82 \%$ & $15.34 \%$ \\
\hline K+W & $100 \%$ & $100 \%$ & $100 \%$ & $100 \%$ & $100 \%$ & $100 \%$ & $100 \%$ & $100 \%$ & $100 \%$ & $100 \%$ \\
\hline DEUDA /PATRIM0NI0 & 0.37 & 0.09 & 0.31 & 0.37 & 0.49 & 0.32 & 0.43 & 0.19 & 0.17 & 0.18 \\
\hline
\end{tabular}

Fuente: Anexo 3 y 4 - (Estado de Ganancias y Pérdidas - Balance General de Sociedad Minera Cerro Verde S.A.A.) presentados en el informe en extenso.

Elaboración: Propia

Los resultados obtenidos por la empresa muestran que la estructura de capital ha sufrido modificaciones muy importantes al igual que el ROE. Se aprecia que maximiza el ROE en el año 2010 llegando al $\mathbf{6 7 . 1 8} \%$ con una estructura adecuada de capital de $\mathbf{7 0 \%}$ de patrimonio y $\mathbf{3 0 \%}$ de deuda. 


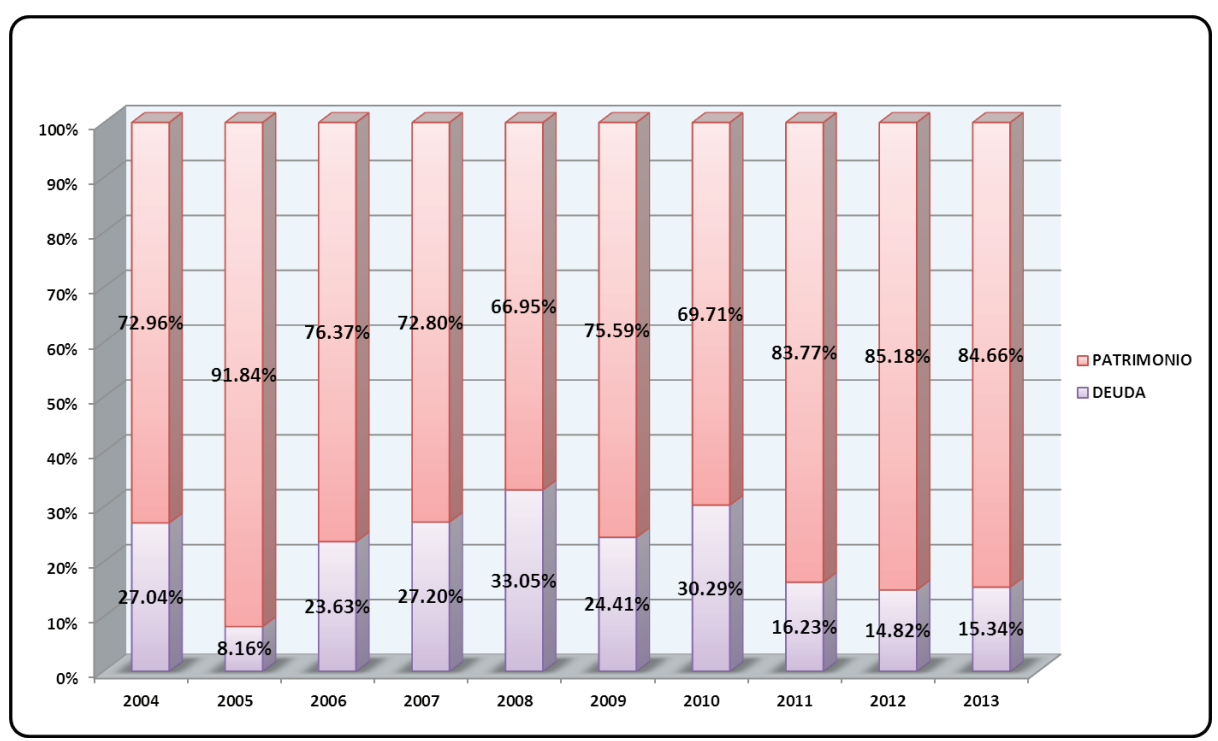

Gráfico $\boldsymbol{n}^{\circ}$ 1. Estructura de Capital - SOCIEDAD MINERA CERRO VERDE S.A.A.

\section{Shougang Hierro Perú S.A.A.}

Con estos datos históricos del activo, pasivo y patrimonio se calculó el ROE y la estructura financiera. Los resultados se muestran a continuación en el Cuadro ${ }^{\circ} 2$ y Gráfico $n^{\circ} 2$.

Los resultados obtenidos por la empresa muestran que la estructura de capital ha sufrido modificaciones muy importantes al igual que el ROE. Se aprecia que maximiza el ROE elo año 2010 llegando al $\mathbf{8 0 . 3} \%$ con una estructura adecuada de capital de $\mathbf{4 4 \%}$ de patrimonio y $\mathbf{5 6 \%}$ de deuda.

Cuadro n' ${ }^{\circ}$. Estructura de Capital de SHOUGANG HIERRO PERÚ S.A.A.

\begin{tabular}{|c|c|c|c|c|c|c|c|c|c|c|}
\hline COEFICIENTE & $\mathbf{2 0 0 4}$ & $\mathbf{2 0 0 5}$ & $\mathbf{2 0 0 6}$ & $\mathbf{2 0 0 7}$ & $\mathbf{2 0 0 8}$ & $\mathbf{2 0 0 9}$ & $\mathbf{2 0 1 0}$ & $\mathbf{2 0 1 1}$ & $\mathbf{2 0 1 2}$ & $\mathbf{2 0 1 3}$ \\
\hline ACTIV0 & 457,430 & 698,776 & 724,771 & 777,336 & $1,039,584$ & $1,135,989$ & $2,309,600$ & $3,948,903$ & $4,103,907$ & $5,271,972$ \\
\hline PASIV0 & 231,940 & 304,229 & 301,244 & 309,567 & 435,723 & 800,059 & $1,290,675$ & $1,287,263$ & $2,994,443$ & $3,911,801$ \\
\hline PATRIMONI0 & 225,490 & 394,547 & 423,527 & 467,769 & 603,861 & 335,930 & $1,018,925$ & $2,661,640$ & $1,109,464$ & $1,360,171$ \\
\hline UTILIDAD NETA & 65,173 & 227,712 & 236,106 & 277,397 & 416,440 & 148,509 & 818,483 & $1,365,870$ & 614,669 & 824,911 \\
\hline ROE & $28.9 \%$ & $57.7 \%$ & $55.7 \%$ & $59.3 \%$ & $\mathbf{6 9 . 0} \%$ & $44.2 \%$ & $80.3 \%$ & $51.3 \%$ & $55.4 \%$ & $60.6 \%$ \\
\hline K & $49.3 \%$ & $56.5 \%$ & $58.4 \%$ & $60.2 \%$ & $\mathbf{5 8 . 1} \%$ & $29.6 \%$ & $44.1 \%$ & $67.4 \%$ & $27.0 \%$ & $25.8 \%$ \\
\hline W & $50.7 \%$ & $43.5 \%$ & $41.6 \%$ & $39.8 \%$ & $\mathbf{4 1 . 9} \%$ & $70.4 \%$ & $55.9 \%$ & $32.6 \%$ & $73.0 \%$ & $74.2 \%$ \\
\hline K+W & $100 \%$ & $100 \%$ & $100 \%$ & $100 \%$ & $100 \%$ & $100 \%$ & $100 \%$ & $100 \%$ & $100 \%$ & $100 \%$ \\
\hline DEUDA/ PATRIM0NI0 & 1.03 & 0.77 & 0.71 & 0.66 & 0.72 & 2.38 & 1.27 & 0.48 & 2.70 & 2.88 \\
\hline
\end{tabular}




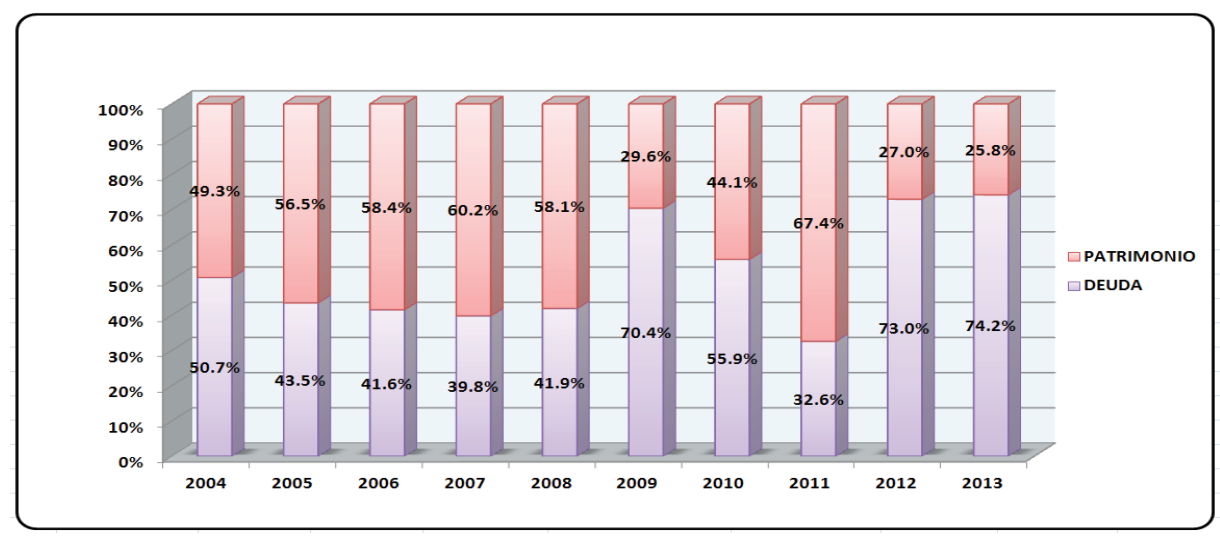

Gráfico $\boldsymbol{n}^{\circ} 2$. Estructura de Capital - SHOUGANG HIERRO PERÚ S.A.A.

\section{Compañía Minera Raura S.A.A.}

Con estos datos históricos del activo, pasivo y patrimonio se calculó el ROE y la estructura financiera. Los resultados se muestran a continuación en el Cuadro n 3 y Gráfico n 3.

Los resultados obtenidos por la empresa muestran que la estructura de capital ha sufrido modificaciones muy importantes al igual que el ROE. Se aprecia que maximiza el ROE en el año 2010 llegando al $79 \%$ con una estructura adecuada de capital de $64 \%$ de patrimonio y $36 \%$ de deuda.

Cuadro n ${ }^{\circ}$ 3. Estructura de Capital de la COMPAÑÍA MINERA RAURA S.A.

\begin{tabular}{|c|c|c|c|c|c|c|c|c|c|c|}
\hline COEFICIENTE & 2004 & 2005 & 2006 & 2007 & 2008 & 2009 & 2010 & 2011 & 2012 & 2013 \\
\hline ACTIV0 & 65846 & 93731 & 228878 & 230057 & 174744 & 166305 & 92983 & 91276 & 87461 & 74809 \\
\hline PASIV0 & 19546 & 30836 & 79519 & 55183 & 59737 & 52329 & 33536 & 32843 & 40661 & 42626 \\
\hline PATRIM0NI0 & 46300 & 62895 & 149292 & 174777 & 114938 & 113896 & 59447 & 58433 & 46800 & 32183 \\
\hline UTILIDAD NETA & 7354 & 17561 & 86639 & 85188 & 20723 & 19727 & 47080 & 15729 & 5337 & -14671 \\
\hline ROE & $15.9 \%$ & $27.9 \%$ & $58.0 \%$ & $48.7 \%$ & $18.0 \%$ & $17.3 \%$ & $79.2 \%$ & $26.9 \%$ & $11.4 \%$ & $-45.6 \%$ \\
\hline K & $70.3 \%$ & $67.1 \%$ & $65.2 \%$ & $76.0 \%$ & $65.8 \%$ & $68.5 \%$ & $63.9 \%$ & $64.0 \%$ & $53.5 \%$ & $43.0 \%$ \\
\hline W & $29.7 \%$ & $32.9 \%$ & $34.8 \%$ & $24.0 \%$ & $34.2 \%$ & $31.5 \%$ & $36.1 \%$ & $36.0 \%$ & $46.5 \%$ & $57.0 \%$ \\
\hline "k" + "w" & 0.30 & 0.33 & 0.35 & 0.24 & 0.34 & 0.31 & 0.36 & 0.36 & 0.46 & 0.57 \\
\hline DEUDA/PATRIM0NIC & 0.42 & 0.49 & 0.53 & 0.32 & 0.52 & 0.46 & 0.56 & 0.56 & 0.87 & 1.32 \\
\hline
\end{tabular}




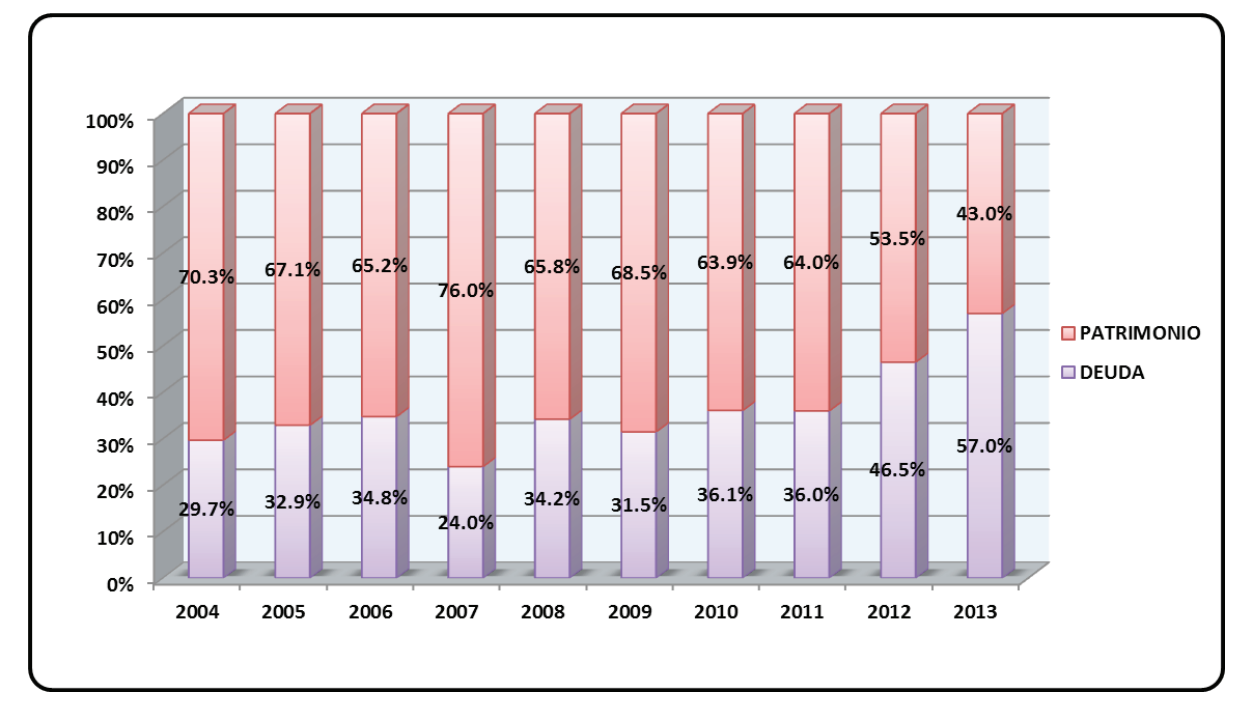

Gráfico $n^{\circ}$ 3. Estructura de Capital -COMPAÑIIA MINERA RAURA S.A.

\section{Análisis de resultados}

Para cada empresa se hizo el análisis que se presenta a continuación. Se toma como ejemplo lo que se presenta en el informe en extenso para la Sociedad Minera Cerro Verde como empresa grande, Shougang Hierro Perú como empresa mediana y Compañía Minera Raura como empresa chica:

\section{Sociedad Minera Cerro Verde S.A.A}

Para el año 2004, esta empresa obtuvo un ROE de $33.00 \%$ superior al ROE promedio de toda la muestra $(21.95 \%)$. a partir de este año presenta una tendencia creciente hasta el 2007 en el que registra un ROE del $55.66 \%$. Como podemos observar en el Gráfico no 4 a partir del 2005, en el que presenta un ROE del $29.40 \%$, empieza a incrementarse llegando a $55.66 \%$. 


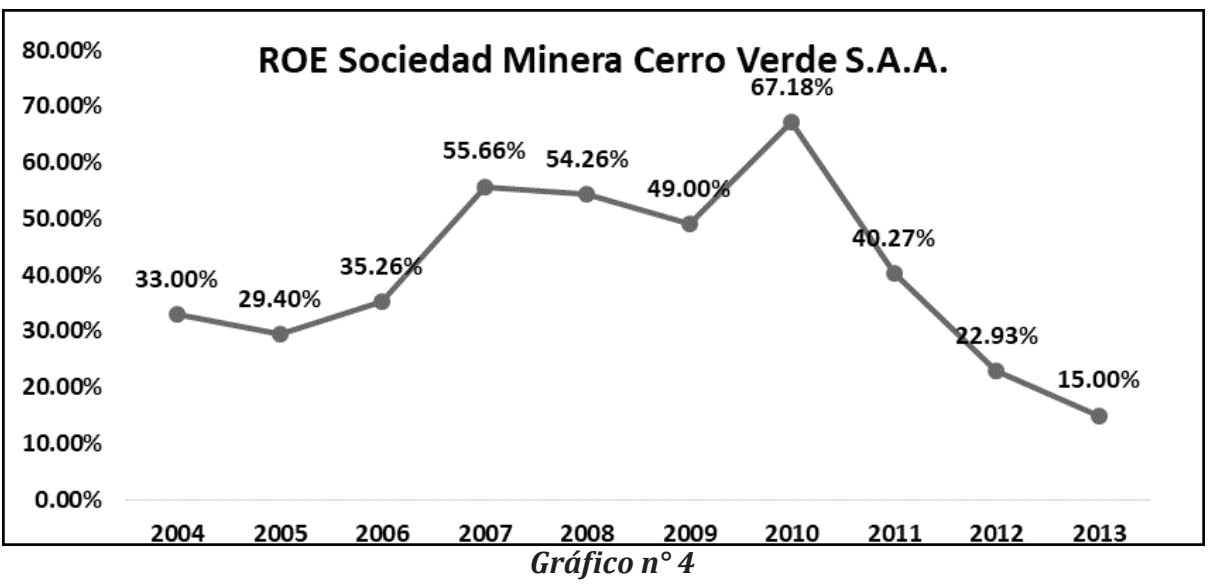

Fuente: Anexo $\mathrm{N}^{\circ} 1$

Elaboración: Grupo de Investigación

Para el año 2008 el ROE de la empresa presenta una caída llegando en el año 2009 al 49.00\% recuperándose en el 2010 llegando a 67.18\% representando el máximo rendimiento del patrimonio obtenido por la empresa en todo el periodo de análisis. A partir del 2011 se observa una caída muy pronunciada llegando el ROE sólo al $15 \%$ muy por debajo del promedio de la muestra.

En los primeros años notaremos que obtuvo una mejora de rentabilidad pasando de un roe de $33 \%$ en el 2004 a un ROE de $54.26 \%$ en el 2008. Podemos observar que del 2004 al 2008 la empresa registró niveles de rentabilidad de su patrimonio por encima del promedio de las empresas de la muestra que fue de $21.95 \%$.

El segundo sub periodo inicia con un ROE del 49\% y llega en el 2010 al 67.18\% constituyéndose en el más alto de todo el periodo de análisis. Sin embargo a partir del 2011 se aprecia una disminución de la rentabilidad del patrimonio que cae hasta $15 \%$ por debajo de la rentabilidad promedio de la muestra.

En conclusión el ROE de la empresa muestra en el periodo de análisis variaciones importantes llegando a maximizar su rentabilidad del patrimonio en el año 2010 con un ROE de $\mathbf{6 7 . 1 8} \%$ y una estructura de capital o financiera del $\mathbf{3 0 . 2 9 \%}$ de deuda y $\mathbf{6 9 . 7 1 \%}$ de patrimonio. 


\section{Shougang Hierro Perú S.A.A.}

Para el año 2004, esta empresa obtuvo un ROE de $28.90 \%$ superior al ROE promedio de toda la muestra (21.95\%). Crece en el año 2005 a $57.70 \%$ y cae ligeramente en el 2006 a 55.70\%. Como podemos observar en el Gráfico $\mathrm{n}$ - 5 a partir del 2007, en el que presenta un ROE del 59.30\%, alcanza en el 2008 una rentabilidad del patrimonio del 69\%, para caer en el 2009 a $44.20 \%$.

En el año 2010 se obtiene el mayor ROE llegando a 80.30\%. Pese a caer en el 2011 a 51.30\%, se recupera en el 2012 llegando a 55.40\%. Cierra el 2013 con un ROE de 60.60\%.

Notaremos que durante los primeros cinco años obtuvo una buena rentabilidad del patrimonio pasando de un ROE de $28.90 \%$ en el 2004 a un ROE de 69\% en el 2008. Podemos observar que del 2004 al 2008 la empresa registró niveles de rentabilidad de su patrimonio por encima del promedio.

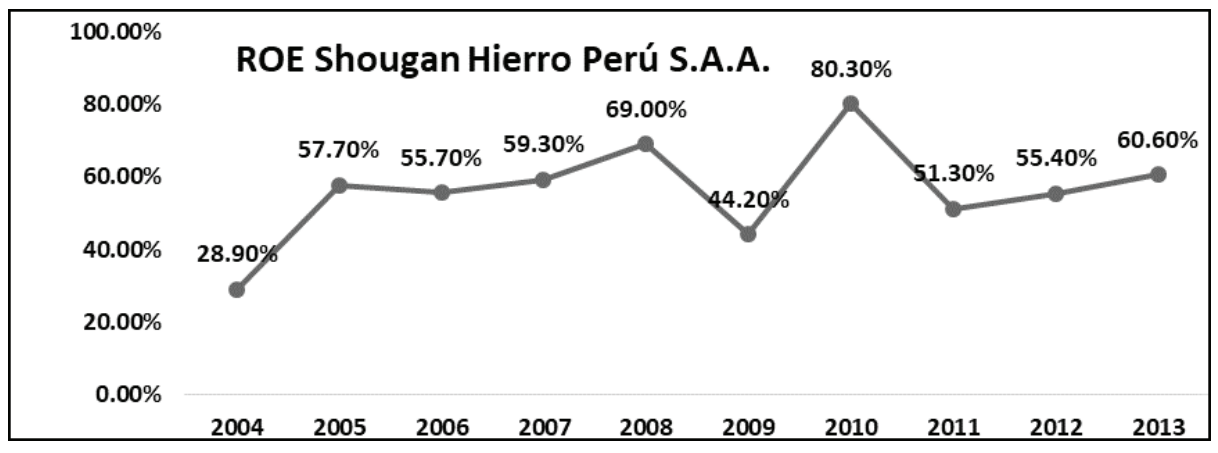

Gráfico $n^{\circ} 5$

Fuente: Anexo $\mathrm{N}^{\circ} 1$

Elaboración: Grupo de Investigación

El segundo sub periodo inicia con un ROE del 44.20\% en el año 2009 y sube en el 2010 al $80.30 \%$. Cae en el 2011 llegando a $51.30 \%$ y crece en el 2012 y 2013 cerrando el ciclo con una rentabilidad del patrimonio del $60.60 \%$. 
En conclusión el ROE de la empresa muestra en el periodo de análisis una tendencia creciente siempre por encima del promedio de la muestra, llegando a maximizar su rentabilidad del patrimonio en el año 2010 con un ROE de $\mathbf{8 0 . 3 0} \%$ y una estructura de capital o financiera del $\mathbf{5 5 . 9 0 \%}$ de deuda y $\mathbf{4 4 . 1 0 \%}$ de patrimonio.

\section{Compañía Minera Raura S.A.A.}

Para el año 2004, esta empresa obtuvo un ROE de $15.90 \%$ inferior al ROE promedio de toda la muestra (21.95\%). Llega en el año 2005 a 27.90\% y en el 2006 a 58\%. Como podemos observar en el Gráfico no 6 a partir del 2007, en el que presenta un ROE del 48.70\%, alcanza en el 2008 una rentabilidad del patrimonio de $18 \%$.

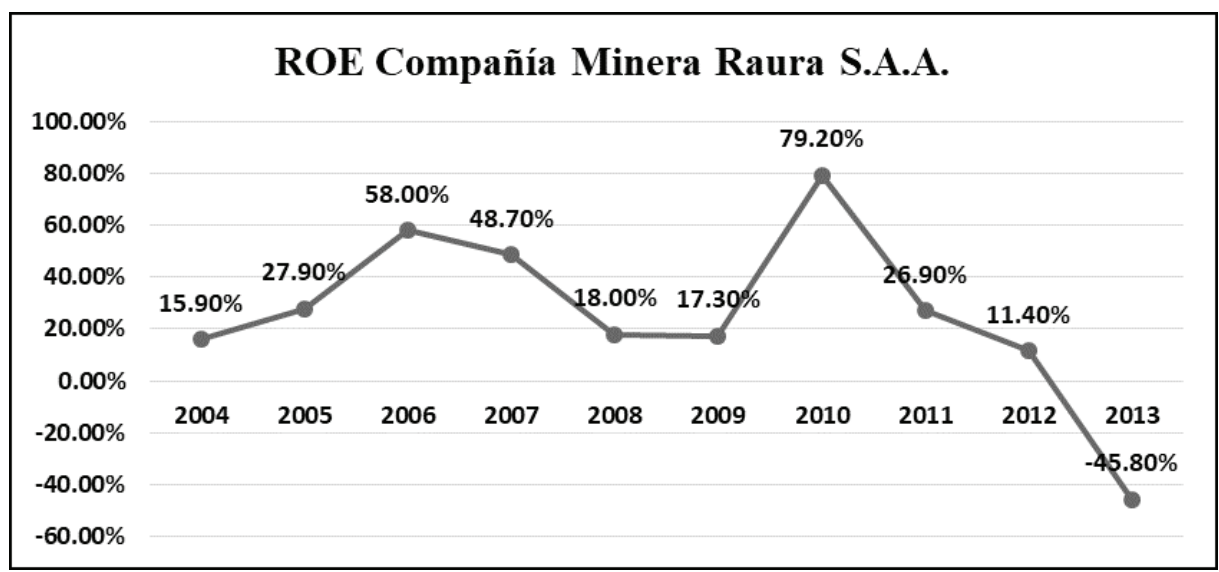

\section{Gráfico $n^{\circ} 6$}

Fuente: Anexo $\mathrm{N}^{\circ} 1$

Elaboración: Grupo de Investigación

En el año 2009 obtiene un ROE de $17.30 \%$, en el 2010 de $79.20 \%$ y en el 2011 de $26.90 \%$. En el 2012 cae al $11.40 \%$ y en el 2013 tiene rentabilidad negativa de $-45.80 \%$.

En los cinco primeros años, notaremos que obtuvo una positiva rentabilidad del patrimonio en los tres primeros años pasando de un ROE de $15.90 \%$ en el 2004 a un ROE de 58\% en el 2006. Podemos observar que del 
2007 al 2008 la empresa registró niveles de rentabilidad de su patrimonio decreciente llegando a $18 \%$.

El segundo sub periodo inicia con un ROE de 17.30\% en el año 2009 y sube pronunciadamente en el 2010 al 79.20\%. A partir del 2011 cae llegando a $26.90 \%$, a $11.40 \%$ en el 2012 y se convierte en negativo en el 2013 llegando a $-45.80 \%$.

En conclusión el ROE de la empresa muestra en el periodo de análisis una tendencia decreciente mostrando en cinco años valores por debajo del promedio de la muestra. Maximiza su rentabilidad del patrimonio en el año 2010 con un ROE de $\mathbf{7 9} \%$ y una estructura de capital o financiera del $\mathbf{3 4 . 8 0} \%$ de deuda y $\mathbf{6 5 . 2 0 \%}$ de patrimonio.

\section{Coclusiones}

1. Después de calcular y analizar el comportamiento de la rentabilidad del patrimonio y su estructura financiera o de capital de cada empresa de la muestra se concluye que el ROE y la estructura financiera han mostrado fluctuaciones con tendencias decrecientes. Esto nos indica que la rentabilidad del capital propio para las empresas del sector han desmejorado en el periodo de análisis, sobre todo en los últimos años, y no se ha mantenido una estructura óptima de capital. Se demuestra la primera hipótesis específica, que dice:

\section{"Las empresas analizadas han presentado una Rentabilidad del Patrimonio fluctuante".}

3. Como se muestra en el Cuadro $\mathrm{n}^{\circ} 4$ una empresa mediana y una chica alcanzaron mejores rentabilidad del patrimonio. Por lo tanto se rechaza la segunda hipótesis específica que dice:

\section{"Las grandes empresas Mineras tuvieron los mejores ren- dimientos de su patrimonio y mejor estructura de capital"}




\section{Recomendaciones}

1. Se recomienda a todas las empresas calcular periódicamente el ROE, para maximizarlo determinando una estructura óptima de capital.

2. Utilizar la metodología planteada dentro del Marco Teórico para determinar la estructura de capital que maximice el ROE de cada empresa.

3. Todas las empresas deben tener como política conseguir una adecuada estructura de capital que les permitan maximizar el ROE.

Cuadro n 4. ROE máximo por empresa en el periodo de análisis 2004-2013

EMPRESA

1. Sociedad Minera Cerro Verde S.A.A.

2. Souther Perú Copper Corporation

3. Compañía Minera Buena ventura S.A.A

4. Volcan Compañía Minera S.A.A.

5. Compañía Minera Milpo S.A.A.

6. Shougang Hierro Perú S.A.A

7. Compañía Minera Atacocha S.A.A.

8. Compañía Minera San Ignacio de Morococha S.A.A.

9. Castrovirreyna Compañía Minera S.A.A

10. Compañía Minera Raura S.A.A.

$\begin{array}{cc}\text { ROE } & \text { TAMAÑO } \\ \text { MAXIMO } & \text { EMPRESA }\end{array}$

$67.18 \% \quad$ Grandes

$49.08 \%$

$42.44 \%$

$52.30 \%$

$41.90 \% \quad$ Medianas

$80.30 \%$

$37.10 \%$

$62.20 \%$

$38.90 \%$

Chicas

$79.20 \%$

Fuente: Anexo $\mathrm{n}^{\circ} 1$

Elaboración: Grupo de investigación. 


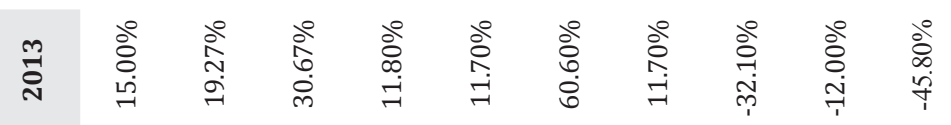

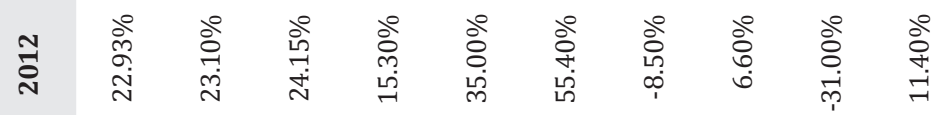

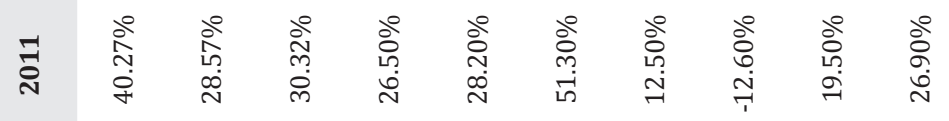

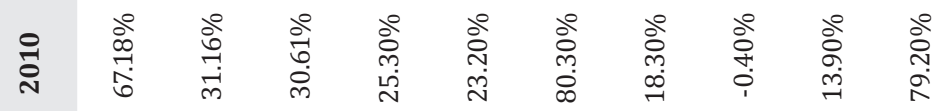

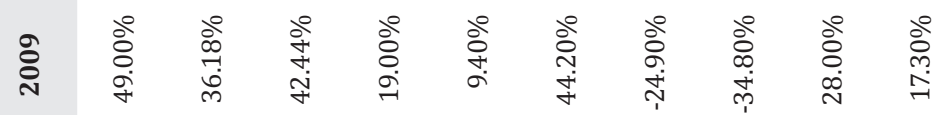

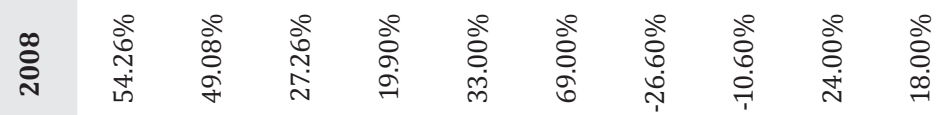

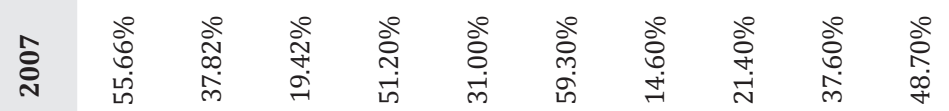

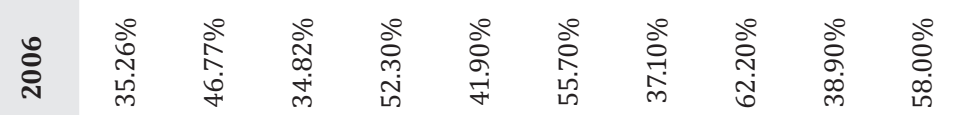

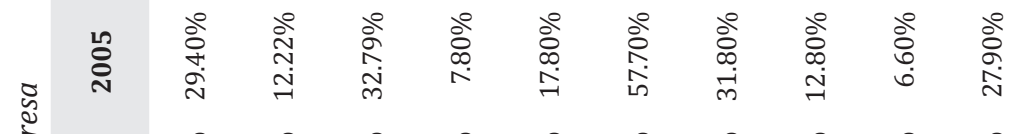

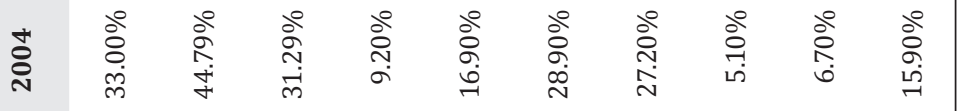

这空

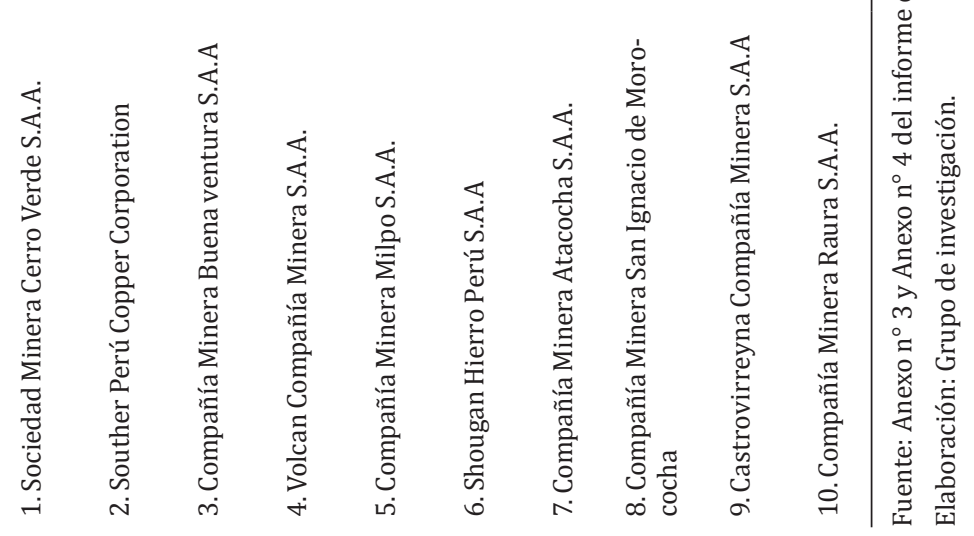




\section{Referencias Bibliográficas}

Córdova Padilla, Marcial (2007). Gerencia Financiera Empresarial. Bogotá, Colombia, ECOE 610p.

Ehrhardt, Michael C. Brigham, Eugene F. (2007). Finanzas Corporativas. México DF, CENEGAGE Learning Editores S.A. 650p.

FORSYTH J.A. (2006). Finanzas Empresariales rentabilidad y valor. Lima, Perú, Editor JAFA 392p.

Pacheco Mexzon, Raimundo Renaun (2014). Gerencia Financiera. Lima, Perú, IIE UNMSM Editor RRPM, 151p.

Pacheco Mexzon, Raimundo Renaun (2010) El EVA y la Creación de Valor De las Empresas Peruanas: Periodo 1999 - 2003. Tesis para optar el grado de Doctor en Economía. Doctorado en Economía, Universidad Nacional Mayor de San Marcos, Perú, 145p.

Ross, Stephen A. Westerfield, Randolph W. Jaffe, Jeffrey. (2005) Finanzas Corporativas. Mc Graw Hill. 967p. 